\title{
DIAGNOSTIC AND THERAPEUTIC STRATEGIES IN PERITONEAL CARCINOMATOSIS FROM BREAST CANCER - LITERATURE MINIREVIEW
}

\author{
Nicolae BACALBASA ${ }^{1,2,3 凶}$, Irina BALESCU ${ }^{4}$, loan SUCIU ${ }^{5}$, Mihaela VILCU ${ }^{2,3}$, \\ Iulian BREZEAN ${ }^{2,3}$, Alin BODOG ${ }^{6}$ \\ ${ }^{1}$ Center of Excellence in Translational Medicine, Fundeni Clinical Institute, Bucharest, Romania \\ 2 „Carol Davila“ University of Medicine and Pharmacy, Bucharest, Romania \\ 3 „Ion Cantacuzino“ Clinical Hospital, Bucharest, Romania \\ ${ }^{4}$ Ponderas Academic Hospital, Bucharest, Romania \\ ${ }^{5}$ Emergency University Hospital, Bucharest, Romania \\ ${ }^{6}$ Oradea Medical University, Romania
}

\begin{abstract}
Peritoneal carcinomatosis with breast cancer origin is an uncommon situation, which usually develops late during the evolution of the disease. The development of such lesions is commonly associated with an aggressive histology (such as lobular carcinoma) and advanced stage at diagnosis and is usually associated with a poor outcome. The positive diagnosis of peritoneal carcinomatosis with breast cancer origin can be sustained only if a biopsy from the tumoral nodules demonstrates the mammary origin of the lesions. In order to improve the outcome of these patients cytoreductive surgery alone or in association with hyperthermic intraperitoneal chemotherapy has been proposed. However, more studies are still needed in order to include the method as part of the therapeutic strategy of these patients.
\end{abstract}

Keywords: breast cancer, peritoneal carcinomatosis, hyperthermic intraperitoneal chemotherapy.

\section{Résumé}

Stratégies diagnostiques et thérapeutiques dans la carcinose péritonéale du cancer du sein - courte revue de la littérature

La carcinose péritonéale d'origine cancéreuse est une situation peu commune, qui se développe généralement tardivement au cours de l'évolution de la maladie. Le développement de telles lésions est généralement associé à une histologie agressive (tel qu'un carcinome lobulaire) et à un stade avancé du diagnostic et est généralement associé à un résultat médiocre. Le diagnostic positif de la carcinose péritonéale d'origine cancéreuse du sein ne peut être maintenu que si une biopsie des nodules tumoraux démontre l'origine mammaire des lésions. Afin d'améliorer l'issue de ces patients, une chirurgie cytoréductionnelle, seule ou en association avec une chimiothérapie hyperthermique intrapéritonéale, a été proposée. Cependant, d'autres études sont encore nécessaires pour inclure 
la méthode dans la stratégie thérapeutique de ces patients.

Mots-clés: cancer du sein, carcinose péritonéale, chimiothérapie hyperthermique intrapéritonéale.

\section{INTRODUCTION}

The majority of malignant tumours developed at the peritoneal level have a metastatic origin, the most commonly incriminated sources being represented by the ovary, colorectal, appendiceal and gastrointestinal primaries ${ }^{1-6}$; however, not only the intra-abdominal malignancies might lead to the apparition of peritoneal carcinomatosis, but also the extra-abdominal ones can produce such lesions. Among the extra-abdominal malignancies leading to peritoneal carcinomatosis, breast and thyroid cancer plays a certain role, ${ }^{7,8}$.

The estimated incidence of peritoneal carcinomatosis from breast cancer remains low, less than 5\% of cases developing such lesions; moreover, it seems that peritoneal involvement occurs later in the progression of the disease ${ }^{9}$, being estimated that the peritoneal malignant contamination produces from the peri-aortic lymph nodes or from the liver metastases?

As for the factors predicting the development of peritoneal carcinomatosis in breast cancer patients, it seems that the most important risk factors are represented by high tumoral grade, lobular invasive histopathological subtype as well as advanced stage at diagnosis?

\section{Diagnosis OF PERITONEAL CARCINOMATOSIS IN BREAST CANCER PATIENTS}

Whenever peritoneal carcinomatosis is suspected in a patient with unknown primary malignancy, biopsy is mandatory in order to establish a positive diagnostic. The biopsy can be taken through surgical approach such as laparotomy or laparoscopy, or, more recently, through a less invasive procedure such as ultrasound guided biopsy or fine needle aspiration. An interesting study focused on this subject originates from Peter et al from Alabama, United States of America ${ }^{10}$. The authors reported a series of four patients submitted to endoscopic ultrasound and biopsy by using trans-gastric or trans-duodenal approach. Among these cases, in one patient breast cancer origin was found for the peritoneal lesions; it was the case of a 54-year-old patient suspected for ovarian cancer presenting for mild ascites, peritoneal carcinomatosis and possible ovarian tumour. The fine needle aspiration obtained through echo-endoscopy raised the suspicion of a poorly differentiated, metastatic carcinoma, so the patient was submitted to total hysterectomy with bilateral adnexectomy and omentectomy, the histopathological studies revealing the presence of tumoral cells at the level of the uterus, both ovaries and cervix, suggestive for metastatic breast cancer ${ }^{10}$.

Another method which can be used to take a biopsy in a female with peritoneal carcinomatosis with unknown origin is represented by the transvaginal ultrasound guided biopsy. In the study conducted by Dadayal et al and published in 2016, the authors included 19 patients suspected of peritoneal carcinomatosis with unknown origin, breast primary being shown in one case; more common origins were represented by ovarian carcinomas (in six cases), endometrial carcinomas (in three cases), cervical or neuroendocrine carcinomas (each in two cases) ${ }^{11}$.

The necessity of peritoneal biopsy in peritoneal carcinomatosis patients, even if the patient had a previous history of breast cancer, is demonstrated by Garg et al in the study which was published in Gynecologic Oncology in 2005 ${ }^{12}$. The authors included in their study 79 patients with peritoneal carcinomatosis which was diagnosed after a median interval of 5.39 years after breast cancer diagnostic. Among the 79 cases, breast cancer origin of the peritoneal lesions was demonstrated only in 19 cases, in the remaining patients the origin of the peritoneal metastases being represented by ovarian cancer or primary peritoneal cancer. Interestingly, 10 out of the 19 patients with peritoneal carcinomatosis with breast cancer origin had a family history of breast cancer; moreover, among the same 19 cases with breast cancer peritoneal metastases, nine patients had previously experienced another recurrence of breast cancer, the peritoneal relapse being in fact the second recurrent event. As for the therapeutic strategy, five out of the 19 cases were successfully submitted to optimal cytoreductive surgery and reported a median overall survival of 34.4 months, significantly higher when compared to suboptimal cytoreduced cases (who experienced an overall survival of only 3.9 months, $p=0.001)^{12}$.

\section{Cytoreductive surgery in association With HYPERTHERMIC INTRAPERITONEAL CHEMOTHERAPY (HIPEC) FOR PERITONEAL CARCINOMATOSIS WITH BREAST CANCER ORIGIN}

Once the benefits of cytoreductive surgery in association with HIPEC were demonstrated in cases with peritoneal carcinomatosis from appendicular, 
colorectal or ovarian origin, certain authors went further and studied the effectiveness of the method in peritoneal carcinomatosis from other primaries ${ }^{1320}$.

An interesting study which investigated the role of cytoreductive surgery in association with HIPEC in patients with peritoneal carcinomatosis from uncommon origins originates from Cardi et al and was published in the World Journal of Surgical Oncology in $2015^{21}$. The study included 28 patients, five of them being submitted to surgery form peritoneal carcinomatosis with breast cancer origin; among the five cases, two patients had been previously diagnosed with lobular carcinomas while the other three cases had been diagnosed with invasive ductal carcinomas. The peritoneal carcinomatosis index ranged between 15 and 24 while the completeness of cytoreduction score was 0 in two cases, 1 in two cases and 2 in one case. Interestingly, the median interval between the initial diagnostic and the development of peritoneal carcinomatosis was 18 years, demonstrating the extremely slow rate of tumoral growth. Moreover, after completing the cytoreduction in association with HIPEC, four out of the five cases reported long-term survival rates, one case being alive 10 years after cytoreduction. These data enabled the authors to demonstrate that the method can be efficient in well selected cases ${ }^{21}$.

The authors described the protocol of HIPEC applied in these cases in a previously published paper; in all cases HIPEC was performed by the closed technique at a temperature of $40^{\circ} \mathrm{C}$, during 60 minutes with cisplatin in a concentration of $75 \mathrm{mg} / \mathrm{m}^{2}$. After this procedure the authors reported that one patient died of disease 56 months later, while the other four cases were still alive and disease free at 13, 45, 74 and 128 months respectively ${ }^{22}$.

In order to determine the potential factors which might influence the evolution of peritoneal carcinomatosis patients after debulking surgery and HIPEC, Passot et al conducted a study in which they investigated the influence of intraperitoneal vascular endothelial growth factor (VEGF) burden ${ }^{23}$. The study included 97 patients with peritoneal carcinomatosis from various origins submitted to debulking surgery between February and October 2012, the most commonly reported origins being colorectal and ovarian - each in 15 cases, followed by pseudomyxoma peritonei and gastric origin; among cases with other origins, one patient was diagnosed with peritoneal metastases from breast cancer origin. The authors measured the intravenous and intraperitoneal levels of VEGF before incision and after abdominal closure (for the intravenous levels) and after ending the resectional phase, after HIPEC, at one and at 24 hours after ending the surgery. The authors demonstrated a significant increase of the intraperitoneal levels of VEGF immediately after debulking surgery, followed by a consistent decrease in the next hours. Moreover, the authors demonstrated that the initial intraperitoneal VEGF levels were significantly associated with the risk of postoperative complications and underlined the fact that preoperative administration of Bevacizumab might decrease these levels and therefore, improve the outcomes of these patients ${ }^{23}$.

An interesting study which aimed to assess the morbidity and mortality of the method if applied in elderly came from Cascales-Campos et al and was published in Surgical Oncology journal in 2016 24 . The study was a multicentric one, conducted in 10 hospitals in Spain and included 85 patients aged over 75 years of age who were submitted to cytoreductive surgery and HIPEC between 2002 and 2014. The median age at the time when entering the study was 77 years while the median peritoneal carcinomatosis index was 12 (range 1-39); when it comes to the origin of the peritoneal lesions, two cases originated from breast cancer. Among the 85 cases, complete cytoreduction was achieved in $79 \%$ of cases, the most commonly performed resections consisting of omentectomy (in $82 \%$ of cases), extensive upper abdominal procedures (in $66 \%$ of cases), diaphragmatic peritonectomy (in $48 \%$ of cases), colonic and parietal resections (in 34\% of cases). After ending the resectional phase, the used HIPEC protocol included Paclitaxel (especially in ovarian cancer patients), Oxaliplatin (for colorectal and appendiceal origin) and Mitomycin C (alone or in association with Doxorubicin). As for the postoperative morbidity, mild complications (grade I-II) were reported in $23.5 \%$ of cases, while moderate to severe complications (grade III-IV) were described in $14.4 \%$ of cases; in the meantime, the 90 days mortality was $5.9 \%$, $3.5 \%$ of patients being dead due to surgery related complications. The three deaths occurred in patients of 81,80 and respectively 75 years of age presenting peritoneal carcinomatosis from colorectal origin (in two cases) and appendiceal origin (in one case) submitted to complete cytoreduction (in two cases) followed by HIPEC with Oxaliplatinum (in the first case) and Mitomycin (in the other two cases). The multivariate analysis demonstrated that complications (irrespective of the degree) were significantly associated with higher than 12 peritoneal carcinomatosis index as well as with the need of perioperative blood transfusion. As for the major complications, the multivariate analysis showed a significant correlation with the preoperative levels of serum albumin, the necessity of performing diaphragmatic peritonectomy procedures as well as the need of blood 
transfusion. There was no correlation in regard with the origin of the primaries ${ }^{24}$.

\section{Conclusions}

Peritoneal carcinomatosis from breast cancer represents an uncommon eventuality. In such cases there is no standardized therapeutic guideline due to the rarity of cases; however, preliminary data have shown that cytoreductive surgery alone or in association with HIPEC might play a role.

\section{Acknowledgements:}

This work was supported by the project entitled „Multidisciplinary Consortium for Supporting the Research Skills in Diagnosing, Treating and Identifying Predictive Factors of Malignant Gynecologic Disorders", project number PN-III-P1-1.2-PCCDI2017-0833.

\section{Compliance with Ethics Requirements:}

„The authors declare no conflict of interest regarding this article"

\section{References}

1. Dohan A, Hobeika C, Najah H, Pocard M, Rousset P, Eveno C. Preoperative assessment of peritoneal carcinomatosis of colorectal origin. J Visc Surg 2018; 155(4): 293-303.

2. Gilly FN, Cotte E, Brigand C, et al. Quantitative prognostic indices in peritoneal carcinomatosis. Eur J Surg Oncol 2006; 32(6): 597-601.

3. Confuorto, G, Giuliano, M E, Grimaldi, A,Viviano, C. Peritoneal carcinomatosis from colorectal cancer: HIPEC? Surg Oncol 2007; 16(Sul 1): S149-S152.

4. Yonemura Y, Endou Y, Sasaki T, et al. Surgical treatment for peritoneal carcinomatosis from gastric cancer. Eur J Surg Oncol 2010; 136(12):1131-1138.

5. Benizri EI, Bereder J M, Rahili A, Bernard J L, Benchimol D. Ascites and malnutrition are predictive factors for incomplete cytoreductive surgery for peritoneal carcinomatosis from gastric cancer. Am J Surg 2013; 205(6):668-673.

6. Hobeika C, Sabbagh C, Najah H, Eveno C. Laparoscopic exploration for peritoneal carcinomatosis: Surgical technique. $J$ Visc Surg 2017; 154(6):430-435.

7. Spratt JS, Edwards M, Kubota T, Lindberg R, Tseng MT. Peritoneal Carcinomatosis: Anatomy, Physiology, Diagnosis, Management, Year Book Medical Publishers, Inc. 1986.

8. Tuthill M, Pell R, Guiliani R, et al. Peritoneal disease in breast cancer: a specific entity with an extremely poor prognosis. Eur J Cancer 2009; 45(12):2146-2149.

9. Diéras V. Peritoneal carcinomatosis and ascites: best practices. Abstracts / The Breast 2017; 36 S1, S19-S76.

10. Peter S, Eltoum I, Eloubeidi MA. EUS-guided FNA of peritoneal carcinomatosis in patients with unknown primary malignancy. Gastrointest Endosc 2009; 70(6):1266-1270.
11. Dadayal G, Weston M, Young A, et al. Transvaginal ultrasound (TVUS)-guided biopsy is safe and effective in diagnosing peritoneal carcinomatosis and recurrent pelvic malignancy. Clin Radio 2016; 171(11):1184-1192.

12. Garg R, Zahurak ML, Trimble EL, Armstrong DK, Bristow RE. Abdominal carcinomatosis in women with a history of breast cancer. Gynecol Oncol 2005; 99(1):65-70.

13. Hanan B, Fonseca LM, Profeta da Luz MM, Lacerda-Filho A, Álvares Cabral MM, da Silva RG. Peritoneal carcinomatosis treated with cytoreductive surgery and intraperitoneal chemotherapy. J Coloproctol (Rio J) 2018; 8; 38(2):172-178.

14. Miailhe G, Arfi A, Mirshahi M, Eveno C, Pocard M, Touboul C. A new animal model for hyperthermic intraperitoneal chemotherapy (HIPEC) in tumor-bearing mice in the treatment of peritoneal carcinomatosis of ovarian origin. $J$ Visc Surg 2018; 155(3):183-189.

15. Castro-Mesta JF, González-Guerreroa JF, Barrios-Sánchez P, Villarreal-Cavazos G. Bases and foundations of the treatment of peritoneal carcinomatosis: Review article. Medicina Universitaria 2016; 18(71):98-104.

16. Laval G, Marcelin-Benazech B, Guirimand F, et al. Recommendations for bowel obstruction with peritoneal carcinomatosis. J Pain Symptom Manage 2014; 48(1), 75-91.

17. Kim KW, Chow O, Parikh K, et al. Peritoneal carcinomatosis in patients with gastric cancer, and the role for surgical resection, cytoreductive surgery, and hyperthermic intraperitoneal chemotherapy. Am J Surg 2014; 207(1), 78-83.

18. Roviello F, Caruso S, Neri A, Marrelli D. Treatment and prevention of peritoneal carcinomatosis from gastric cancer by cytoreductive surgery and hyperthermic intraperitoneal chemotherapy: overview and rationale. Eur J Surg Oncol 2013; 39(12), 1309-1316.

19. Lorimier G, Linot B, Paillocher N, et al. Curative cytoreductive surgery followed by hyperthermic intraperitoneal chemotherapy in patients with peritoneal carcinomatosis and synchronous resectable liver metastases arising from colorectal cancer. Eur J Surg Oncol 2017; 43(1), 150-158.

20. Sardi A, Jimenez WA, Nieroda C, Sittig M, Macdonald R, Gushchin V. Repeated cytoreductive surgery and hyperthermic intraperitoneal chemotherapy in peritoneal carcinomatosis from appendiceal cancer: analysis of survival outcomes. Eur J Surg Oncol 2013; 39(11), 1207-1213.

21. Cardi M, Sammartino P, Mingarelli V, et al. Cytoreduction and HIPEC in the treatment of "unconventional“ secondary peritoneal carcinomatosis. World J Surg Oncol 2015; 13 305.

22. Cardi M, Sammartino P, Framarino ML, et al. Treatment of peritoneal carcinomatosis from breast cancer by maximal cytoreduction and HIPEC: a preliminary report on 5 cases. Breast 2013; 22(5), 845-849.

23. Passot G, Bakrin N, Garnier L, et al. Intraperitoneal vascular endothelial growth factor burden in peritoneal surface malignancies treated with curative intent: the first step before intraperitoneal anti-vascular endothelial growth factor treatment? Eur J Cancer 2014; 50(4), 722-730.

24. Cascales-Campos PA, Lopez-Lopez V, Munoz-Casares FC, et al. Morbidity and mortality outcomes after cytoreductive surgery and hyperthermic intraperitoneal chemotherapy in patients aged 75 years and over: Spanish group of peritoneal cancer surgery (GECOP) multicenter study. Surg Oncol 2016; 25(2), 111-116. 